\title{
A Simple Algorithm for Drawing Large Graphs on Small Screens
}

\author{
Karlis Kaugars $^{1}$, Juris Reinfelds ${ }^{1}$, Alvis Brazma ${ }^{2}$ \\ 1 New Mexico State University, Las Cruces NM 88003, USA \\ 2 Institute of Mathematics and Computer Science, University of Latvia, Riga, Latvia
}

\begin{abstract}
Viewing a large graph in limited display space has traditionally been accomplished using either reduced scale rendering of the graph or by attaching scrollbars to a view window which shows only a small portion of the entire graph. Recent work, however, has concentrated on integrating a locally detailed view with a globally scaled view. We present an algorithm for constructing a view which smoothly integrates local detail and global context in a single view window and describe user interaction with such a display.
\end{abstract}

\section{Introduction}

The aim of this work is to develop a graph layout visualization where the user can view one or more areas of interest in relative detail, simultaneously not losing an overview of the entire graph structure and the relative positions of the areas of interest.

To achieve this goal, we need a transformation which maps an area of any size to the size of the view window in a way that chosen parts can be mapped linearly and the rest of the graph is compressed to fit the screen. Furthermore, we need a tranformation such that there is a smooth transition between the linear and compressed sections of the graph so that visual continuity is maintained.

Such a transformation can be implemented by a monotonic, continuous function which is almost linear in some central part, asymptotic to a constant at $\pm \infty$ and smoothly passes from one into the other. A simple function that meets these requirements is the arctangent, hence the name of our algorithm, Compressed ArcTangent Graph (CATGraph).

\subsection{Related Work}

Sarkar and Brown [3] present a method called the Graphical Fisheye Views of Graphs, where the layout is transformed by both a distance based degree of interest function and a priori knowledge about the relative importance of nodes. The transformation is, however, limited to a single point of interest.

Misue, et al. [2] have independently discovered the same transformation which we apply. They dismiss the transformation as inappropriate for their display as it tends to compress areas at great distances to single points and does not preserve orthogonal ordering of nodes in the polar case. 


\section{The CATGraph Algorithm}

Assuming an unbounded graph layout $(-\infty<x<+\infty)$ with a point of interest at $x_{i}$ and a radius of interest $r$ around $x_{i}$, the conversion of original-graph layout to viewed-graph layout is accomplished by the application of the function

$$
f(x)=\arctan \left(\frac{0.5}{r}\left(x-x_{i}\right)\right)
$$

independently for the $\mathrm{x}$ and $\mathrm{y}$ coordinates. The transformation is applied to the center point of each node and the four surrounding corner points. This transformation yields a display similar to the one depicted in Fig. 1.
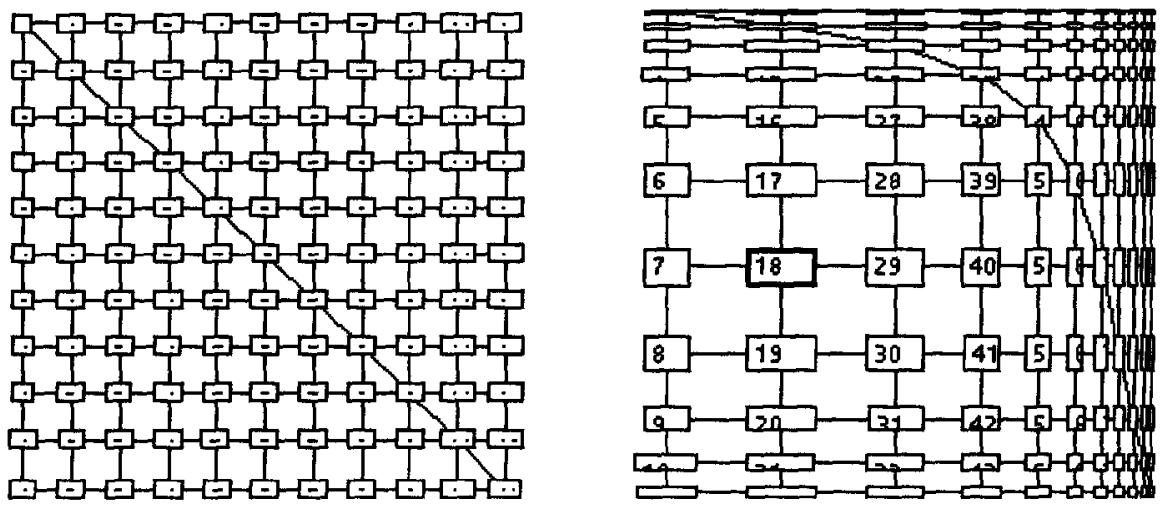

Fig. 1. Results of applying the CATGraph algorithm to a graph. The image on the left is the linearly scaled graph. The image on the right has a single point of interest centered on the node labeled 18.

Multiple points of interest can be introduced by converting each display point of the graph once for every point of interest and then taking the mathematical average of the transformed points as the display location.

The result of $(1)$ is a graph layout which is always scaled to the interval $\pm \frac{\pi}{2}$. While helpful when displaying graphs without known minimum and maximum values, in practice the graph should be scaled to the size of the output window.

Since the function is applied independently in the $\mathrm{x}$ and $\mathrm{y}$ directions, the transformed graph layout displays the width of nodes on a line of constant $\mathrm{x}$ at $x_{i}$ at the same scale as any node centered at $\left(x_{i}, y_{i}\right)$, and the height of nodes on a line of constant y at $y_{i}$ at the same scale as any node centered at $\left(x_{i}, y_{i}\right)$. When several points of interest are selected, the function creates ghost points of interests at the intersections of these lines, such as node 15 and 73 in Fig. 2. This difficulty can be avoided by using a polar transformation of the graph layout. If the point to be converted is located at the point of interest, it is transformed to 
$(0,0)$. Otherwise, the polar transformation first converts the location of point $(x, y)$ to a distance $d$ and angle $\theta$ from a point of interest at $\left(x_{i}, y_{i}\right)$ by application of

$$
d=\sqrt{\left(x-x_{i}\right)^{2}+\left(y-y_{i}\right)^{2}}
$$

and

$$
\theta=\arctan \left(\frac{y-y_{i}}{x-x_{i}}\right)
$$

The distance $d$ is then transformed by the arctan function utilizing the radius of interest $r$

$$
d_{t}=\arctan \left(\frac{0.5}{r} d\right)
$$

and finally transformed back to a rectangular coordinate system by

$$
x_{t}=d_{t} \cos \theta
$$

and

$$
y_{t}=d_{t} \sin \theta \text {. }
$$

As before, multiple points of interest can be applied to a graph layout by taking the arithmetic mean of the converted points. The application of polar conversion with multiple points of interest is illustrated in Fig. 2.
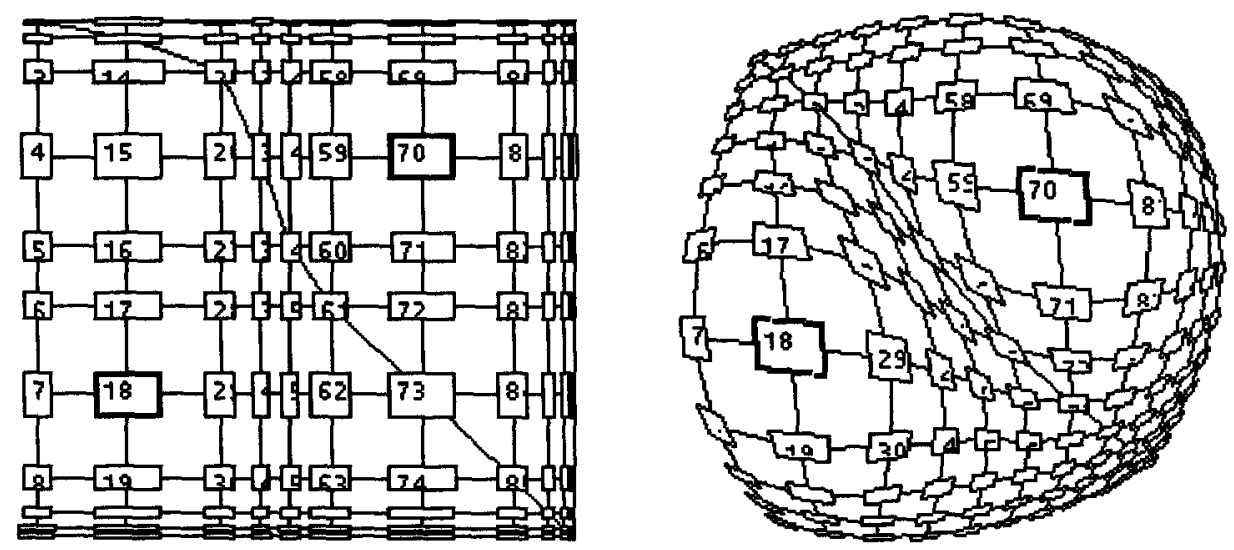

Fig. 2. Multiple points of interest. Both images have points of interest on the nodes labeled 18 and 70 . The image on the left is converted using the rectangular transformation, while the image on the right is transformed using the polar transformation. 


\section{Usability Considerations}

Interaction with the transformed graph can be accomplished with simple mousebased methods based on node display location. Selecting a node can be done in view space by finding the nearest displayed center point of a node and selecting the node this center point represents. A similar technique can be applied for locating the nearest point of interest. Creating, deleting, and moving points of interest can therefore be accomplished with simple mouse-based interactions. Scaling the degree of interest can be accomplished by displaying a slider at the selected point of interest.

Viewing the contents of nodes is done in place by decreasing the radius of interest almost zero and scaling the transformed graph to fit the entire viewing window. Scaling the transformed view has the consequence of transforming the node to a size larger than it appeared in the original layout, thereby allowing considerable space for the display of contents.

Changes to the radius of interest and locations of points of interest can have a dramatic effect on the size and positioning of nodes. The changes should be animated. The animation allows the user to better comprehend the effect of modifications to points of interest.

Because the combination of points of interest is additive, clustered points of interest tend to dominate isolated points of interest. We are currently attempting to develop a combination function which allows for automatically selecting degrees of interest based on proximity of other points of interest. We are also investigating the use of non-point source transformations which might alleviate the problem.

\section{Summary}

The CATGraph display allows the integration of local and global views in a single, size-limited viewing window. It allows the display of multiple points of interest and display of node contents in place. We have noted interaction techniques appropriate to the display and suggested future work which may overcome the shortcomings of the transformation.

\section{References}

1. Kaugars, K.; BASS and CAT: Two Fisheye Views of Trees; Master's Thesis; New Mexico State University; Las Cruces, NM, USA; May, 1992.

2. Misue, K., et al.; Layout Adjustment and the Mental Map. Institute for Social Information Science Research Report ISIS-RR-94-6E; Fujitsu Laboratories Ltd.; Miyamoto, Japan.

3. Sarkar, M., Brown M.H; Graphical Fisheye Views of Graphs; in Proceedings of CHI '91; Monterey, CA, USA; May , 1992; 83-91. 